\section{MS28 P01}

Thin film delamination study during in situ compressive testing by Scanning micro X-ray Diffraction P. Goudeau ${ }^{\mathrm{a}}$, G. Geandier ${ }^{\mathrm{a}}$, P.-O. Renault ${ }^{\mathrm{a}}, \mathrm{N}$. Tamura $^{\mathrm{b}}$, C. Coupeau ${ }^{\mathrm{a}}$, F. Foucher ${ }^{\mathrm{a}},{ }^{\mathrm{a}} L M P$, University of Poitiers, France. ${ }^{\mathrm{b}} A L S-L B N L$, Berkeley, USA. E-mail: pgoudeau@univ-poitiers.fr

\section{Keywords: metallic coatings, mechanical stresses, buckling}

The understanding of the mechanical properties of coated materials is a key factor in a number of technological applications. In particular, the delamination of compressed thin films is one of most limiting factor for the structural performance of the material and presents various interesting problems in physics and mechanics.

The delamination patterns result from relaxation phenomenon of high residual compressive stresses in the thin film mainly due to the deposition process and involve the propagation, from an initial buckle, of cracks at the film/substrate interface. They have been extensively investigated in various experimental and theoretical studies [1-5]. However, only a few experimental set-ups have been developed to measure the buckle growth dynamics. In fact, the additional compressive stress can be produced in the adherent thin film by using an experimental apparatus which allows the in-situ observation by AFM of the surface during deformation. Buckling patterns generated during the stress experiments are then described and discussed taking advantage of the high resolution offered by the atomic force microscopy (AFM). It has been observed that buckling structures may evolve from straight-sided wrinkles to either worm-like or varicose patterns when the applied stress is released. The formation of these two structures from the initial straightsided wrinkles has been characterized. In particular, it has been found that one of the driving forces of delamination is the relaxation of the stresses along the longitudinal axis of the initial wrinkles. In the case of bubbles, the film does not recombine with the substrate; in situ picosecond acoustic experiments [2] would allow studying the adhesion between film and substrates in this region of interest. Finite element simulations have been done in order to investigate the delamination evolution $[4,5]$. Confrontations with in situ stress mapping measurements have to be achieved for validating and then improving these simulations.

In this work, we proposed to use the Scanning X-Ray microdiffraction technique developed at the ALS to perform in-situ spatially resolved stress measurements [1, 3] during a compression test on a film/substrate set. These measurements are expected to provide new insights on the first stage of the delamination process. Preliminary experiments have been done at ALS on gold thin films deposited on LiF substrates.

[1] P. Goudeau, P. Villain, N. Tamura, H. A. Padmore, Applied Physics Letters, 2003, 83, 51.

[2] C. Coupeau, P. Goudeau, L. Belliard, M. George, N. Tamura, F. Cleymand, J. Colin, B. Perrin, J. Grilhé, Thin Solid Films, 2004, 469-470, 221.

[3] P. Goudeau, N. Tamura, G. Parry, J. Colin, C. Coupeau, F. Cleymand, H. Padmore, Mater. Res. Soc. Symp. Proc., 2005, 875, 1.

[4] G. Parry, J. Colin, C. Coupeau, F. Foucher, A. Cimetière, J. Grilhé, Acta Materiala, 2005, 53, 441. F. Foucher, C. Coupeau, J. Colin, A. Cimetière, J. Grilhé, Phys. Rev. Letters, 2006, 97, 1.

\author{
MS28 P02 \\ Synchrotron Laue micro-diffraction: a new beam line \\ project at SOLEIL for phase identification and \\ mechanics of materials Philippe Goudeau ${ }^{\mathrm{a}}$, Olivier \\ Thomas ${ }^{\mathrm{b}},{ }^{\mathrm{a}} L M P$, University of Poitiers, France. ${ }^{\mathrm{b}}$ TECSEN, \\ University of Marseille, France \\ E-mail: pgoudeau@univ-poitiers.fr
}

Keywords: 2D mapping, grain orientation, in grainstrains

Micro-focused x-ray beams from 1 down to less than 0.1 micron in size have been one of the real success stories of 3rd generation synchrotron x-ray machines (SR), thanks to a large panel of focusing devices for hard and soft x-rays [1-5]. There is clearly a rapidly growing need for very small x-ray beams $-10 \mathrm{~nm}$ is theoretically possible which allow for non-destructive local scale measurements of structure and chemistry. This need encompasses many different scientific fields: Microelectronics and microsystems, Metallurgy and mechanics, Environmental and earth sciences, Art and archaeology, Life sciences and soft condensed matter. In all these different research fields one would ideally like to get information on a local scale of the structure, the chemical composition and the local atomic environment. This implies performing at the submicron scale: XRD, XRF, EXAFS, XANES. However, the case of XRD technique is specific in the sense that the recorded signal does not only depend on the beam size but also on the ratio of the beam size to the grain size. If this ratio is large enough one is left with powder diffraction when using monochromatic x-ray beam (MB) and the diffraction information is an average over the size of the beam. On the other hand if the beam size over grain size ratio is small one gets single crystals diffraction. It is thus possible to obtain intra grain structural information. Transmission configuration with hard $\mathrm{x}$-rays is done using either MB or white beam (WB) XRD with generally energy dispersive mode. For the proposed beam line, the working geometry is reflection. It is important to realize, however, that recording a significant number of diffraction spots from a single crystal requires either a movement of the sample under the beam (goniometry) or the use of a polychromatic incoming beam (Laue diffraction) combined with a two-dimensional area detector such as CCD type. Since there are no available goniometers with a sphere of confusion (SOC) radius smaller than the micrometer $\mathrm{x}$-ray probe size it is thus necessary to use WB. Details concerning the different applications of the technique may be found for instance at the Advanced Light Source (ALS) web site address, this synchrotron source being close enough to the French facility SOLEIL. This beam line project is unique in Europe since there is almost nothing in European countries in terms of fully dedicated WB $\mu$ XRD beam line except the BM32 project at ESRF $(10 \%$ of the full beam line time dedicated to microfocus WB during 3 years). The Scientific Advisory Committee (SAC) of SOLEIL approved the preliminary beam line project APS (document available at CnanoNO web site) in November 2005 and the SOLEIL Council decided in July 2006 to build this additional beam line and to provide $1 / 4$ of the total beam line budget which will be completed by external funds (not yet found).

[1] F. Pfeiffer, C. David, M. Burghammer, C. Rieckel, T. Salditt, Science, 2002, 297, 230.

[2] C. G. Schroer, B. Lengeler, Phys. Rev. Letters, 2005, 94, 054802 . 